\title{
“SPRING-BACK” CLOSURE ASSOCIATED WITH OPEN-DOOR CERVICAL LAMINOPLASTY
}

Authors:

${ }^{1}$ Hai Qiang Wang, MD, ${ }^{2}$ Kin Cheung Mak, MBBS, FRCSEd, FHKCOS, FHKAM(Ortho), ${ }^{2}$ Dino Samartzis, DSc, ${ }^{3}$ Tarek El-Fiky, MD, ${ }^{2}$ Yat Wa Wong, MBBS, FRCSEd, FHKCOS, FHKAM(Ortho), ${ }^{1}$ Zhuo Jing Luo, MD, ${ }^{2}$ Xin Kang, MD, ${ }^{2}$ Wai Yeun Cheung, MBBS, FRCSEd, FHKCOS, FHKAM(Ortho), ${ }^{2}$ Keith D.K. Luk, MBBS, MCh(Ortho), FRACS, FRCSEd, FRCS(Glas), FHKCOS, FHKAM(Ortho), ${ }^{2}$ Kenneth M.C. Cheung, MBBS(UK), MD, FRCS(England), FHKCOS, FHKAM(Ortho)

Affiliations: $\quad{ }^{1}$ Department of Orthopaedics, Xijing Hospital, Fourth Military Medical University, Xi'an, China; the ${ }^{2}$ Department of Orthopaedics and Traumatology, University of Hong Kong, Pokfulam, Hong Kong, SAR China; and the ${ }^{3}$ Department of Orthopaedic Surgery, Alexandria University, Alexandria, Egypt

Disclosure: $\quad$ The authors have no financial or competing interests to disclose.

Key Words: $\quad$ cervical; laminoplasty; open-door; spring-back; closure; complication; outcome

Correspondence: Kenneth M.C. Cheung

Department of Orthopaedics \& Traumatology

Queen Mary Hospital

Professorial Block, $5^{\text {th }}$ Floor

102 Pokfulam Road

Hong Kong, SAR, China

Tel: +85228554254

Fax: +852 28174392

Email: ken-cheung@hku.hk 


\section{STRUCTURED ABSTRACT}

Background Context: Spring-back complication after open-door laminoplasty as described by Hirabayashi is a well-known risk, but its definition, incidence, and associated neurological outcome remains unclear.

Objective: To investigate the incidence and the neurological consequence of spring-back closure following open-door laminoplasty.

Study Design: A retrospective radiographic and clinical review.

Outcome Measures: Lateral cervical spine x-rays were evaluated. Anteroposterior diameter (APD) of the vertebral canal of C3 to C7 was measured. Spring-back was defined as loss of APD on follow-up in comparison to immediate post-operative canal expansion. The loss of the end-on lamina silhouette with consequent re-appearance of the lateral profile of the spinous processes was also assessed to verify the presence of spring-back. Spring-back closure was classified based on whether the collapse was total or partial, and whether all the operated levels or only a subset has collapsed (i.e. complete vs. partial closure, segmental closure vs total construct closure). Neurological status was documented using the Japanese Orthopaedic Association (JOA) score.

Methods: Thirty consecutive patients who underwent open-door laminoplasty from 1995 to 2005 at a single institution with a minimum follow-up of 2 years were assessed. They were all operated upon using the classic Hirabayashi technique. Radiographic outcomes were assessed independently by two individuals. 
Results: Sixteen males and 14 females with an average follow-up of 5 years (range; 2 - 12 years) were included. Of these patients, 24 had cervical spondylotic myelopathy and 6 had ossification of the posterior longitudinal ligament. Spring-back closure was found in 3 patients (10\%) and 7 out of 117 laminas (6\%) within 6 months of the operation, which was further confirmed by CT and MRI imaging. All spring-back closures were partial segmental closures. Gender and age were not significant factors related to spring-back ( $p>0.05)$. The mean JOA score on follow-up was 12.5 , with a recovery rate of $40 \%$. All patients with spring-back and available JOA data exhibited postoperative neurologic deterioration. Of the 3 patients with spring-back, 2 patients underwent revision surgery whereas 1 declined.

Conclusions: Spring-back closure occurred in 10\% of our patients at or before 6 months following surgery. The incidence of spring-back by level (i.e. 117 laminas) was 6\%, mainly occurring at the lower cervical spine. All spring-back closures were partial segmental closures, most commonly involving C5 \& C6. Postoperative neurological deficit was associated with spring-back closure therefore surgeons should adopt pre-emptive surgical measures to prevent the occurrence of such a complication. 


\section{INTRODUCTION}

Cervical laminoplasty is an established procedure for treatment of cervical myelopathy. ${ }^{1-5}$ Throughout the years, various laminoplasty techniques have been described. However, open-door laminoplasty, first described by Hirabayashi ${ }^{6}$ in the 1970 s, is commonly performed. The procedure allows widening of the spinal canal without permanently removing the dorsal cervical elements, thereby addressing the disadvantages of laminectomy (i.e. spinal cord vulnerability, cervical kyphosis, etc). Although successful outcomes have been associated with this procedure, various postoperative complications may occur, such as axial neck pain, neck stiffness, nerve root palsy, loss of lordosis, and "spring-back" closure of the elevated lamina. ${ }^{3,7-11}$

Collapse or spring-back of the open-door, whereby the lamina closes back on the spinal cord, has been a salient concern. Such a complication would negate the benefit of the procedure, leading to immediate re-stenosis of the canal. Since the initial description of opendoor laminoplasty, reports of spring-back closure emerged as the procedure was more widely adopted. Although there have been numerous reports on the long-term results of open-door laminoplasty, ${ }^{9-10,12-18}$ little is known of the actual definition, the detailed incidence, and the attendant neurological outcomes associated with spring-back closure. As such, the following study proposes a definition of spring-back closure as well as addresses the incidence and clinical outcomes associated with spring-back complication based on a review of patients who underwent open-door laminoplasty at a single institution. 


\section{MATERIALS AND METHODS}

Between January 1995 and December 2005, 30 consecutive patients who underwent open-door laminoplasty at the ----- (location masked for review) ---- were reviewed retrospectively. The diagnosis of cervical myelopathy was made based on clinical signs and symptoms, with corresponding levels of stenosis confirmed by magnetic resonance imaging (MRI) of the cervical spine.

\section{Surgical Technique}

The operative levels were dependent upon the neurological status and the radiological findings. The open-door laminoplasty technique described by Hirabayashi and Satomi ${ }^{11}$ was employed. Since the C3-C5 spinous processes were typically very short, they did not necessitate shortening or cutting, thereby preserving the supra- and interspinous ligaments. Normally, the tips of the C6 and/or C7 spinous processes were shortened because they were too long in comparison to the other spinous processes and if not shortened the posterior elements may be pushed back after wound closure. Both the hinge- and open-side gutters were created with a high-speed burr. The open-side gutter was completed with a Kerrison rongeur, and the laminae elevated by gently pushing the spinous processes toward the hingeside. Stay sutures were inserted between the base of the spinous process and the facet joint capsule at each level. The neck was immobilized with a rigid collar for 3 weeks.

\section{Radiographic Assessment}

Pre- and postoperative lateral cervical spine x-rays were obtained for all patients. Standardization was achieved by ensuring $150 \mathrm{~cm}$ film-to-tube distance and centering on the C4 body. Radiographs were digitized and entered into a DICOM Picture Archiving \& 
Communication (PAC) system. Visualization and measurements were taken using a Radworks 5.1 (Applicare Medical Imaging BV, Zeist, The Netherlands) system.

Postoperative x-rays were taken on the day following the operation, and subsequently during out-patient follow up. Follow up was usually scheduled at 2-4 weeks after hospital discharge, and at 3-6 month intervals thereafter in the first 2 years.

The anteroposterior diameter (APD) of the vertebral canal for each level was measured independently by 2 spine orthopaedic surgeons. Pre-operative APD was measured using Wolf's method (Figure 1A). ${ }^{19}$ Post-operative APD was measured from the middle of the posterior border of the vertebral body to the anterior cortex of the elevated lamina (Figure 1B), which is fully compatible with the pre-operative APD measurement.

Spring-back complication was defined as loss of initial elevation of the lamina as determined on the postoperative follow-up radiographs. A classification scheme was proposed and illustrated in Table 1. In the lateral cervical x-ray, a closed lamina would also appear as substitution of the oval or teardrop silhouette of the elevated lamina by the shape of the remaining spinous process profile. Significant APD decrease was corroborated by the loss of the oval or teardrop silhouette on the lateral imaging. Thus, for any patient with springback, they could be partial or complete closure (for any one level), while the involvement maybe segmental (only a subset of levels operated is/are affected) or total construct (all levels involved). While there may be other combinations of spring-back, some levels are less likely involved and the intact spinous processes with their supraspinous and interspinous ligaments may not allow them as such.

\section{Neurological Outcome Assessment}

Neurological status was appraised using the Japanese Orthopaedic Association (JOA) score for assessing cervical myelopathy. ${ }^{20} \mathrm{~A}$ trained occupational therapist performed all the 
JOA score assessments for each patient. The JOA score recovery rate was used to reflect the degree of postoperative recovery of normal function. .-3,9-11,21 $^{-1}$

\section{Statistical Analysis}

All data was collected and coded upon a spreadsheet. SPSS vr. 14 (Chicago, IL, USA) statistical software was utilized to perform the statistical analyses. Descriptive and frequency statistics were performed of various data parameters. Inter-class coefficient was utilized to assess inter-rater reliability. Reliability scores of less than $0.79,0.80-0.89$, and greater than 0.90 were regarded as poor, good, and excellent, respectively. ${ }^{22}$ Descriptive and frequency statistics were performed as well as Mann-Whitney U, Chi-Square, and Fisher's Exact tests where appropriate. A p-value less than 0.05 was considered statistically significant.

\section{RESULTS}

There were 16 males (53.3\%) and 14 females (47.7\%), with a mean age of 63.6 years ( \pm SD: 12.7 years; range: $24-83$ years). Of these patients, 24 had cervical spondylotic myelopathy and 6 patients had ossification of posterior longitudinal ligament. The mean follow-up period was 4.9 years ( \pm SD: 2.8 years; range: $2-12$ years). Two cases had concomitant non-iatrogenic interbody fusion of the cervical spine (i.e. congenital or spontaneous). In total, 117 laminas were elevated. The mean number of elevated laminas were 3.9 ( \pm SD: 0.9) for each patient. Levels of laminoplasty were as follows: C3-C6 in 17 patients (56.7\%), C3-C7 in 6 patients (20\%), C3-C5 in 3 patients (10\%), C3-C4 in 2 patients (6.7\%), C4-C6 in 1 patient (3.3\%), and C4-C5 in 1 patient (3.3\%). The reason for the reduced length of laminoplasties in some patients were because they were involved in a pilot trial 
comparing anterior versus posterior surgery, and care was taken to ensure the decompression was equivalent in the anterior versus posterior group.

\section{Radiographic Assessment}

A good to high inter-rater reliability was noted in the assessment of spring-back (>0.89). Spring-back was noted in 3 out of 30 patients (10\%), affecting 7 out of 117 elevated laminas (6\%) (Figure 1C-G). The order of involvement was as follows: C3 (0/28; 0\%), C4 (1/30; 3.3\%), C5 (3/28; 10.7\%), C6 (3/24; 12.5\%), and C7 (0/6; 0\%) (Figure 2).

In all three patients, spring-back was noted as partial closure and occurred within 6 months of surgery. All were partial segmental closures. Furthermore, closure was at contiguous levels among those multi-level collapses. Spring-back did not occur in any of the 2 patients with anterior column fusion. Gender and age were not significant factors related to spring-back closure $(\mathrm{p}>0.05)$.

\section{Neurological Outcomes}

The average preoperative JOA score was 9.5 ( \pm SD: 3.4). By 6 months, the JOA score was 12.06 ( \pm SD: 3.1) with a recovery rate of 35\% using the method of calculation proposed

by Hirabayashi et al. ${ }^{1}$ At final follow up, the postoperative JOA score was 12.5 ( \pm SD: 3.2 ), producing a recovery rate of $40 \%$. All patients with spring-back and available JOA data exhibited neurological deficit (Table 2).

In the 3 patients who exhibited radiological spring-back, 2 had a decreased JOA score and thus required a laminectomy to decompress the spinal cord. Among these 2 patients, one patient's radiological spring-back predated the clinical manifestation more than 8 years. For the third patient, the JOA score was 12 pre-operatively and the subsequent change in 
neurological status did not affect the JOA score significantly; thus, decided against further surgical intervention.

\section{DISCUSSION}

Open-door laminoplasty as initially reported by Hirabayashi ${ }^{6}$ is commonly used for posterior decompression in patients with cervical myelopathy. ${ }^{1-5}$ Its major advantage over more recently described methods is the speed of the procedure and the relative low cost as no implants are used. Because of the relatively insecure method of fixing the opened lamina, spring-back has always been a concern. However, there is relative paucity of information regarding the details of the "spring-back" phenomenon. ${ }^{3,7-11}$ According to Hirabayashi and Satomi ${ }^{6}$ in their seminal report of this procedure, the open-door was maintained by stay sutures placed "through the deep muscles and capsules around the facets of the hinge side" and "tied around the bases of the spinous processes through the yellow ligaments".

In our study, we aimed to define spring-back clearly, in both morphologic x-ray features and correlating it to the APD. Furthermore, from the APD measurements of individual laminoplasty levels, we began to appreciate that each elevated level may be evaluated as an independent event though multi-lamina afflictions tend to affect contiguous levels. An elevated lamina will have an end-on profile in the lateral plain radiograph appearing as an oval or teardrop silhouette. ${ }^{10}$ When this disappeared and was replaced by the lateral profile of the spinous process, spring-back closure was suggested. By comparing serial x-rays, this method can be quite accurate. However, utilizing the APD measurement, a 4-mm decrease corresponded to spring-back when relying upon the morphologic assessment of the lateral cervical x-rays. 
We further defined spring-back based on two parameters (Table 1): whether at a particular level the lamina collapsed to its pre-operative position (i.e. complete vs. partial closure of a specific level) and whether all the operated levels suffered lamina collapse (i.e. total construct closure). Not a single patient from our series had total construct closure. Furthermore, of the involved laminas, all were partial closures. In other words, not all levels are affected equally. Specifically, C5 and C6 had closure rates of 10\% and 12.5\%, respectively, while C4 had 3.3\%.

Using the definition we proposed, 10\% (3 out of 30) of patients had spring-back, whereby $6 \%$ (7 out of 117) of all elevated laminae had such closure. This was despite the use of stay sutures in every patient. The complication rate per patient was significantly higher than that reported by Satomi et $a l^{18}(1.5 \%)$ or Chiba et $a l^{10}(3.7 \%)$, though their definition of spring-back remains unclear and Chiba only measured the anteroposterior canal diameter at C4. The variation between our incidence rate in comparison to previously reported accounts of spring-back may be attributed to our detailed classification of how spring-back is defined. On the other hand, our series represented the initial phase after adoption of the Hirabayashi procedure, and experience probably played a role in the spring-back rate.

In their very first 15 patients, Hirabayashi and Satomi ${ }^{11}$ reported "several cases" that experienced spring-back. Subsequent to using stay sutures, these patients “almost always maintained the canal open successfully”. As such, the authors attributed spring-back to the absence of stay sutures. In a subsequent report, Satomi et al ${ }^{17}$ noted 3 out of 80 patients (1.5\%) with laminar closure. Chiba et al ${ }^{10}$ noted that 1 out of 27 patients (3.7\%) undergoing cervical laminoplasty developed postoperative myelopathy due to closure of the elevated lamina. However, these reports did not provide detailed definition, or temporal relationship between spring-back closure and neurological deterioration, and the method of identifying spring-back closure may have been limited. A recent prospective randomized study 
evaluating the clinical outcome of single open-door vs. double open-door laminoplasties noted one patient out of 17 which had spring-back at 3 months and required an anterior surgery. Matsumoto et al ${ }^{23}$ provided a very precise method of evaluating spring-back, the canal-to-body ratio. By dividing the APD of the spinal canal by that of the width of the corresponding vertebral body, magnification error was reduced. However, there is some controversy in the value of using canal-to-body ratios given the intrinsic variability of the 2 parameters.

The authors suggest a much simpler method of screening for re-stenosis following cervical laminoplasty. According to Hirabayashi et al, ${ }^{3}$ utilizing stay sutures for cervical single door expansion laminoplasty, a mean of 3 to 5-mm APD widening was noted and that 4-mm was the optimal APD for neurological recovery. By using 4-mm in APD as our cutoff, we were able to identify individuals who were likely to suffer from the clinical consequence of spring-back. Only 3 patients suffered clinical deterioration and all had spring-back by our definition. Indeed, pre-operative planning by way of CT and MRI for 2 of the patients who agreed for revision surgery confirmed re-stenosis, primarily at those levels that demonstrated APD loss of 4-mm or more. The third patient declined further surgery and due to resource limitations did not have these investigations. Arguments against this method would include variations in APD related to gender and height. In 2004, Lim and Wong ${ }^{24}$ showed that this variation in sagittal diameter was less among ethnic Chinese, and further argued that the lateral radiographic sagittal diameter of the canal was a better method for diagnosing cervical canal stenosis than the Torg-Pavlov ratio, which is essentially a canal-to-body ratio. Further larger scale prospective studies are needed to define the predictive value in terms of likelihood of clinical deterioration, but our method proves to be a very practical tool for identifying patients who need closer scrutiny, if not further investigation and treatment. 
Cervical motion has been studied in normal and abnormal subjects, as well as postoperative patients. In the normal cervical spine, the majority of motion of the subaxial spine occurs at the lower regions. ${ }^{25-28}$ Most studies examining the post-laminoplasty movement of the cervical spine showed a decreased range of motion from $17-50 \%{ }^{29}$ Miyazaki et al ${ }^{30}$ reviewed the relative contribution of each level at various stages of degeneration, and his numbers indicated that until end-stage degeneration C5 and C6 still contribute significantly to the flexion-extension arc of the cervical spine. Also, a study of the axial rotation of the postlaminoplasty cervical spine showed that angular movement was similar to that before the operation. ${ }^{31}$ Taking these two studies into account, a plausible explanation for the increased risk of spring-back at C5 and C6 may be that even in the setting of a laminoplasty, motion is still relatively concentrated in these areas.

The JOA recovery rate of $40 \%$ found in our study was relatively low when compared to other studies, which showed upwards of $50 \% .{ }^{1,10,18}$ This may be explained by Satomi et $a$ 's $^{18}$ finding that the recovery rate was adversely affected once the patient was 60 years or older. The average age of our patients was 64 years, while that of Satomi et al ${ }^{18}$ and Chiba et $a l^{10}$ were 57 years and 55 years, respectively.

Numerous studies have reported spring-back complication after laminoplasty, but the degree of closure and the temporal relationship between collapse of the lamina and neurological deterioration was not elucidated. While a slight amount of collapse (i.e. 1-mm or less) is a common occurrence, in our patients this was not usually associated with neurological deterioration. However, using our radiographic definition and correlation with APD measurements, we noted that 4-mm collapse was eventually associated with a decreased JOA score.

Various methods have been proposed to prevent lamina closure. Apart from modification of Hirabayashi et al's original technique, others have proposed the use of 
interposition bone graft, mini-plates, and suture anchors. ${ }^{29,32-35}$ From our study, we noted that spring-back was most common at C5 and C6, with low occurrence at C3 and C7. We have also shown that significant spring-back (i.e. loss of the teardrop silhouette or APD decrease of 4-mm or more) will cause neurological compromise. While Hirabayashi et al's technique of suturing the elevated laminae to the facet capsules is an improvement over having no attachment at all (based on their first publication), our study suggests further augmentation may be necessary. Therefore, if resources are limited, mini-plates or suture anchors like Mitek, may be considered at C5 \& C6, in addition to non-absorbable stay sutures, as a more secure and stable means to affix the elevated laminae.

Although our study noted interesting observation regarding spring-back closure following open-door cervical laminoplasty, various limitations were present. For one, our study consisted of a relatively small sample size and was retrospective in nature. However, since the concept and occurrence of spring-back was largely neglected in previous report, we feel that our study can provide some added information regarding such a potential complication. Furthermore, another potential limitation of the study is the lack of advanced imaging for every patient to correlate with the plain radiographic assessments. However, advanced imaging was performed in the spring-back cases, which confirmed the complication. Nevertheless, additional larger, prospective studies are needed to further verify our findings and expand on our definition of spring-back.

\section{CONCLUSIONS}

Spring-back is not an infrequent complication, oftentimes associated with neurological compromise. In our study population, the overall incidence of spring-back was 
noted as $10 \%$, mainly occurring at C5 and C6. Moreover, spring-back may occur within the first 6 months following surgery and may potentially be associated with neurologic compromise. Furthermore, spring-back closure was noted to be partial and usually not involving the entire laminoplasty construct. Given that significant spring-back is associated with neurological compromise, we suggest augmenting only at the high risk levels, i.e. C5 and C6. In addition, our paper is the first to propose a detailed description of spring-back closure following open-door laminoplasty and, in doing, hopefully enable a simple yet reliable method for defining, screening and communicating this phenomenon. 


\section{ACKNOWLEDGEMENT}

We would like to thank Daniel YT Fong and Florence Mok for their help in the statistical analyses, and Karlen Law for her work on the JOA score assessment. 


\section{REFERENCES}

1. Hirabayashi K, Miyakawa J, Satomi K, et al. Operative results and postoperative progression of ossification among patients with ossification of cervical posterior longitudinal ligament. Spine 1981;6:354-64.

2. Hirabayashi K, Toyama Y, Chiba K. Expansive laminoplasty for myelopathy in ossification of the longitudinal ligament. Clin Orthop Relat Res 1999:35-48.

3. Hirabayashi K, Watanabe K, Wakano K, et al. Expansive open-door laminoplasty for cervical spinal stenotic myelopathy. Spine 1983;8:693-9.

4. Iwasaki M, Okuda S, Miyauchi A, et al. Surgical strategy for cervical myelopathy due to ossification of the posterior longitudinal ligament: Part 2: Advantages of anterior decompression and fusion over laminoplasty. Spine 2007;32:654-60.

5. Kawahara N, Tomita K, Shinya Y, et al. Recapping T-saw laminoplasty for spinal cord tumors. Spine 1999;24:1363-70.

6. Hirabayashi K. [Expansive open-door laminoplasty for cervical spondylotic myelopathy]. Jpn J Surg 1978;32:1159-63.

7. Hosono N, Sakaura H, Mukai Y, et al. The source of axial pain after cervical laminoplasty-C7 is more crucial than deep extensor muscles. Spine 2007;32:2985-8.

8. Baba H, Maezawa Y, Furusawa N, et al. Flexibility and alignment of the cervical spine after laminoplasty for spondylotic myelopathy. A radiographic study. Int Orthop 1995;19:116-21.

9. Seichi A, Takeshita K, Ohishi I, et al. Long-term results of double-door laminoplasty for cervical stenotic myelopathy. Spine 2001;26:479-87. 
10. Chiba K, Ogawa Y, Ishii K, et al. Long-term results of expansive open-door laminoplasty for cervical myelopathy--average 14-year follow-up study. Spine 2006;31:2998-3005.

11. Hirabayashi K, Satomi K. Operative procedure and results of expansive open-door laminoplasty. Spine 1988;13:870-6.

12. Azuma S, Seichi A, Ohnishi I, et al. Long-term results of operative treatment for cervical spondylotic myelopathy in patients with athetoid cerebral palsy: an over 10year follow-up study. Spine 2002;27:943-8; discussion 8.

13. Hale JJ, Gruson KI, Spivak JM. Laminoplasty: a review of its role in compressive cervical myelopathy. Spine J 2006;6:289S-98S.

14. Iwasaki M, Kawaguchi Y, Kimura T, et al. Long-term results of expansive laminoplasty for ossification of the posterior longitudinal ligament of the cervical spine: more than 10 years follow up. J Neurosurg 2002;96:180-9.

15. Kimura I, Shingu H, Nasu Y. Long-term follow-up of cervical spondylotic myelopathy treated by canal-expansive laminoplasty. J Bone Joint Surg Br 1995;77:956-61.

16. Ogawa Y, Toyama Y, Chiba K, et al. Long-term results of expansive open-door laminoplasty for ossification of the posterior longitudinal ligament of the cervical spine. J Neurosurg Spine 2004;1:168-74.

17. Satomi K, Nishu Y, Kohno T, et al. Long-term follow-up studies of open-door expansive laminoplasty for cervical stenotic myelopathy. Spine 1994;19:507-10.

18. Satomi K, Ogawa J, Ishii Y, et al. Short-term complications and long-term results of expansive open-door laminoplasty for cervical stenotic myelopathy. Spine $J$ 2001;1:26-30. 
19. Wolf BS, Khilnani M, Malis L. The sagittal diameter of the bony cervical spinal canal and its significance in cervical spondylosis. J Mt Sinai Hosp N Y 1956;23:283-92.

20. Yonenobu K, Abumi K, Nagata K, et al. Interobserver and intraobserver reliability of the japanese orthopaedic association scoring system for evaluation of cervical compression myelopathy. Spine 2001;26:1890-4; discussion 5.

21. Kawai S, Sunago K, Doi K, et al. Cervical laminoplasty (Hattori's method). Procedure and follow-up results. Spine 1988;13:1245-50.

22. Liu A, Schisterman EF, Wu C. Multistage evaluation of measurement error in a reliability study. Biometrics 2006;62:1190-6.

23. Blackley HR, Plank LD, Robertson PA. Determining the sagittal dimensions of the canal of the cervical spine. The reliability of ratios of anatomical measurements. $J$ Bone Joint Surg Br 1999;81:110-2.

24. Lim J-K, Wong H-K. Variation of the cervical spinal Torg ratio with gender and ethnicity. The Spine Journal 2004;4:396-401.

25. Fielding JW. Cineroentgenography of the normal cervical spine. J Bone Joint Surg Am 1957;39-A:1280-8.

26. Fielding JW. Normal and Selected Abnormal Motion of the Cervical Spine from the Second Cervical Vertebra to the Seventh Cervical Vertebra Based on Cineroentgenography. J Bone Joint Surg Am 1964;46:1779-81.

27. Ordway NR, Seymour RJ, Donelson RG, et al. Cervical flexion, extension, protrusion, and retraction. A radiographic segmental analysis. Spine 1999;24:240-7.

28. Panjabi M, Dvorak J, Duranceau J, et al. Three-dimensional movements of the upper cervical spine. Spine 1988;13:726-30.

29. Steinmetz MP, Resnick DK. Cervical laminoplasty. Spine J 2006;6:274S-81S. 
30. Miyazaki M, Hong SW, Yoon SH, et al. Kinematic analysis of the relationship between the grade of disc degeneration and motion unit of the cervical spine. Spine 2008;33:187-93.

31. Sugimoto Y, Tanaka M, Nakanishi K, et al. Assessing range of cervical rotation after laminoplasty using axial CT. J Spinal Disord Tech 2007;20:187-9.

32. Chen HC, Chang MC, Yu WK, et al. Lateral mass anchoring screws for cervical laminoplasty: preliminary report of a novel technique. J Spinal Disord Tech 2008;21:387-92.

33. Lee JY, Hanks SE, Oxner W, et al. Use of small suture anchors in cervical laminoplasty to maintain canal expansion: a technical note. J Spinal Disord Tech 2007;20:33-5.

34. O'Brien MF, Peterson D, Casey AT, et al. A novel technique for laminoplasty augmentation of spinal canal area using titanium miniplate stabilization. A computerized morphometric analysis. Spine 1996;21:474-83; discussion 84.

35. Yang SC, Yu SW, Tu YK, et al. Open-door laminoplasty with suture anchor fixation for cervical myelopathy in ossification of the posterior longitudinal ligament. J Spinal Disord Tech 2007;20:492-8. 


\section{FIGURE LEGENDS}

Figure 1: Radiographs of a patient who experienced spring-back with anteroposterior diameter (APD) measurements (mm). (A) Preoperative x-ray showing APD measured using Wolf's method, from the middle of the posterior border of the vertebral body to the anterior border of the lamina. (B) Postoperative x-ray illustrating the oval or teardrop silhouette of the elevated lamina and the increased APD measurements. (C) Spring-back closure occurred at C5 \& C6, whereby the end-on cortical silhouette of the elevated lamina is replaced by the lateral profile of the spinous process. Note the corresponding decrease in APD of more than 4-mm at these two levels. (D, E) CT and (F,G) MRI scans demonstrated and confirmed spring-back closure of C5 and C6.

Figure 2: The incidence of spring-back per cervical spine level. 

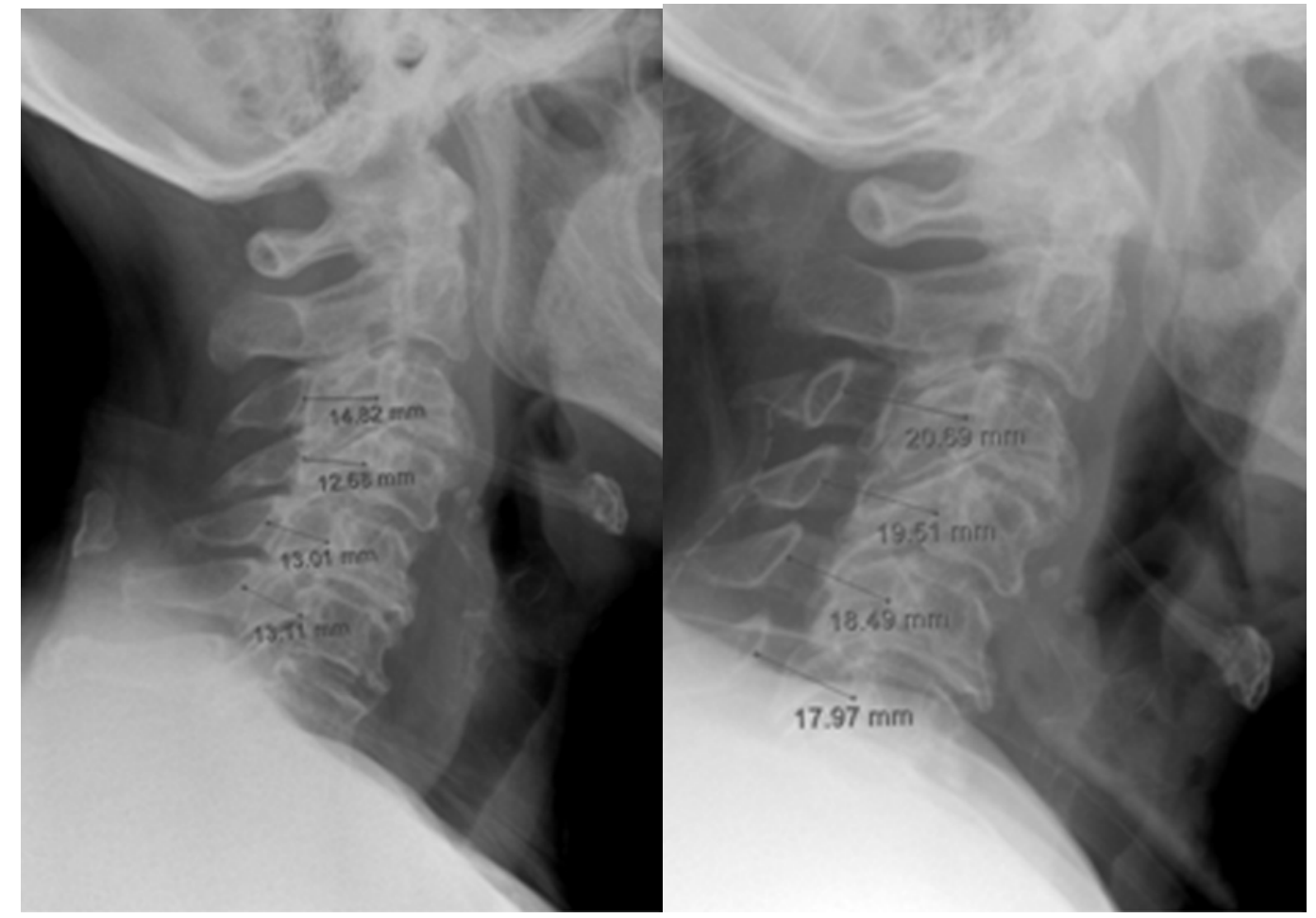

Figure $1 \mathrm{a}, \mathrm{b}$

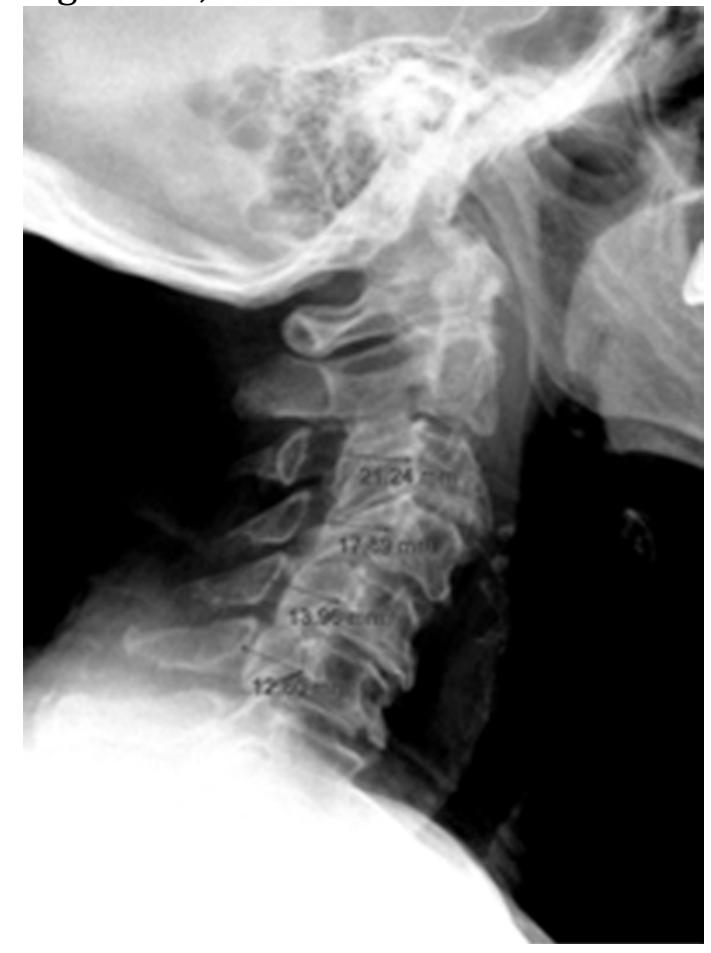

Figure 1c 


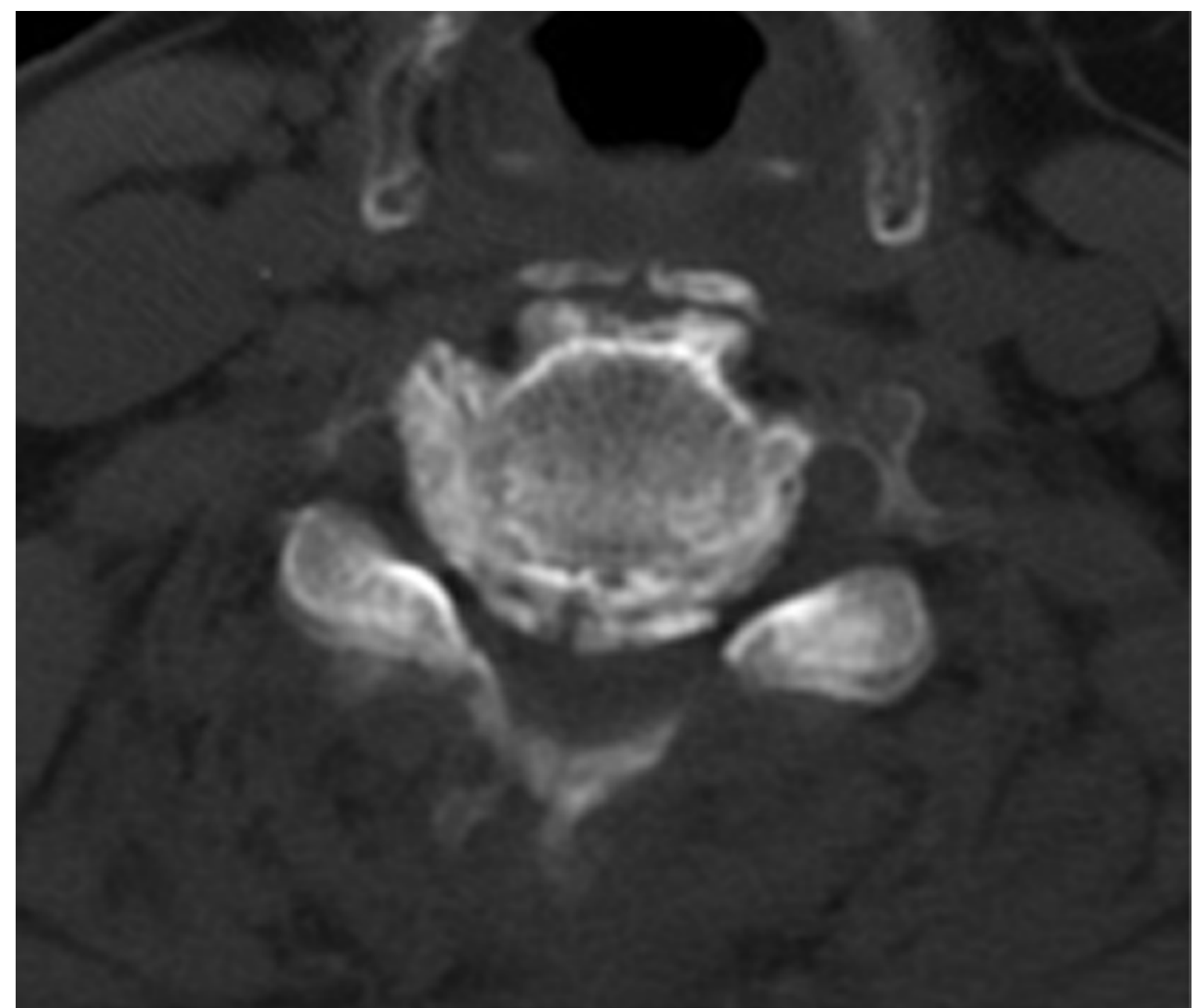

Figure 1d 


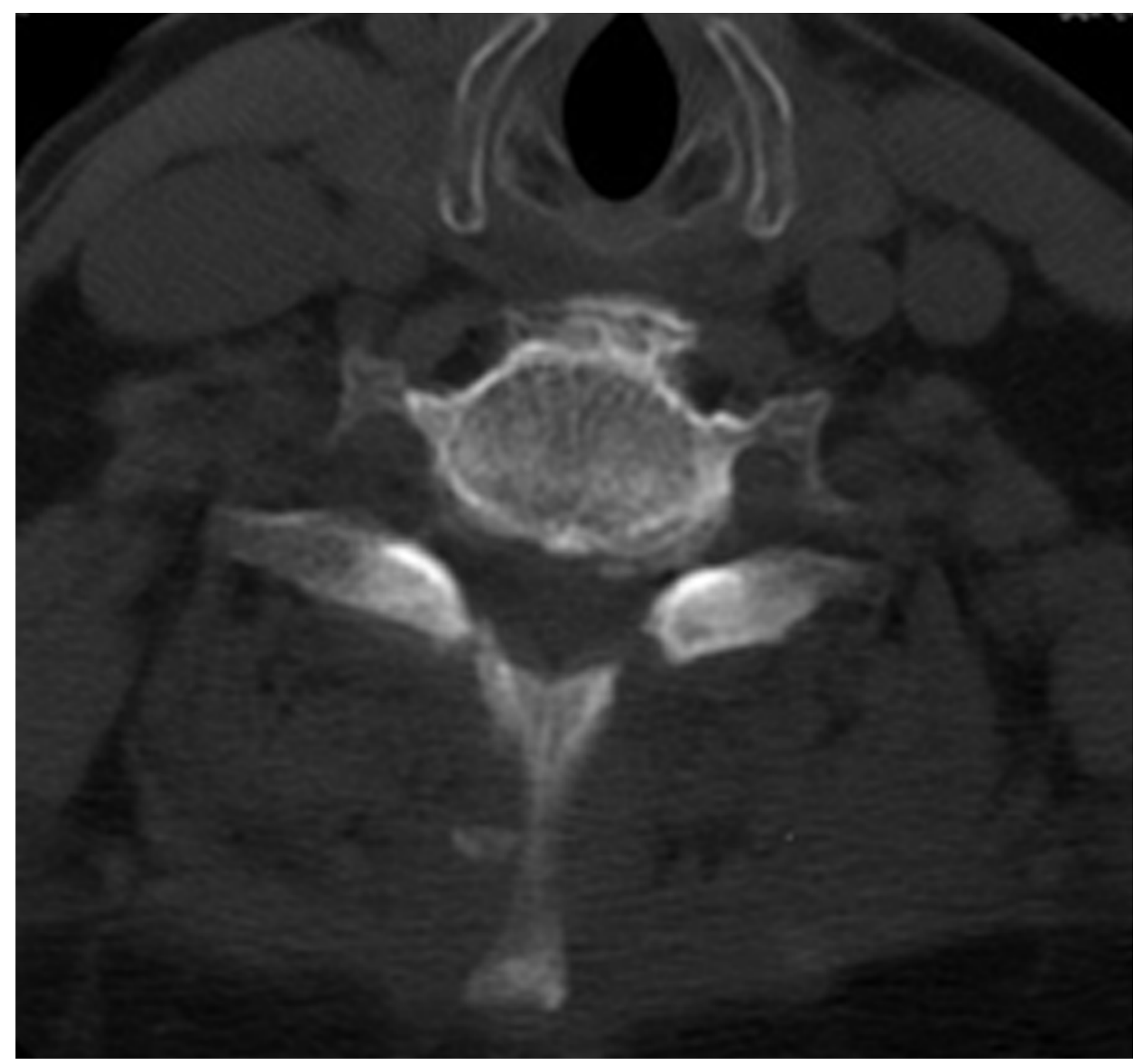

Figure 1e 


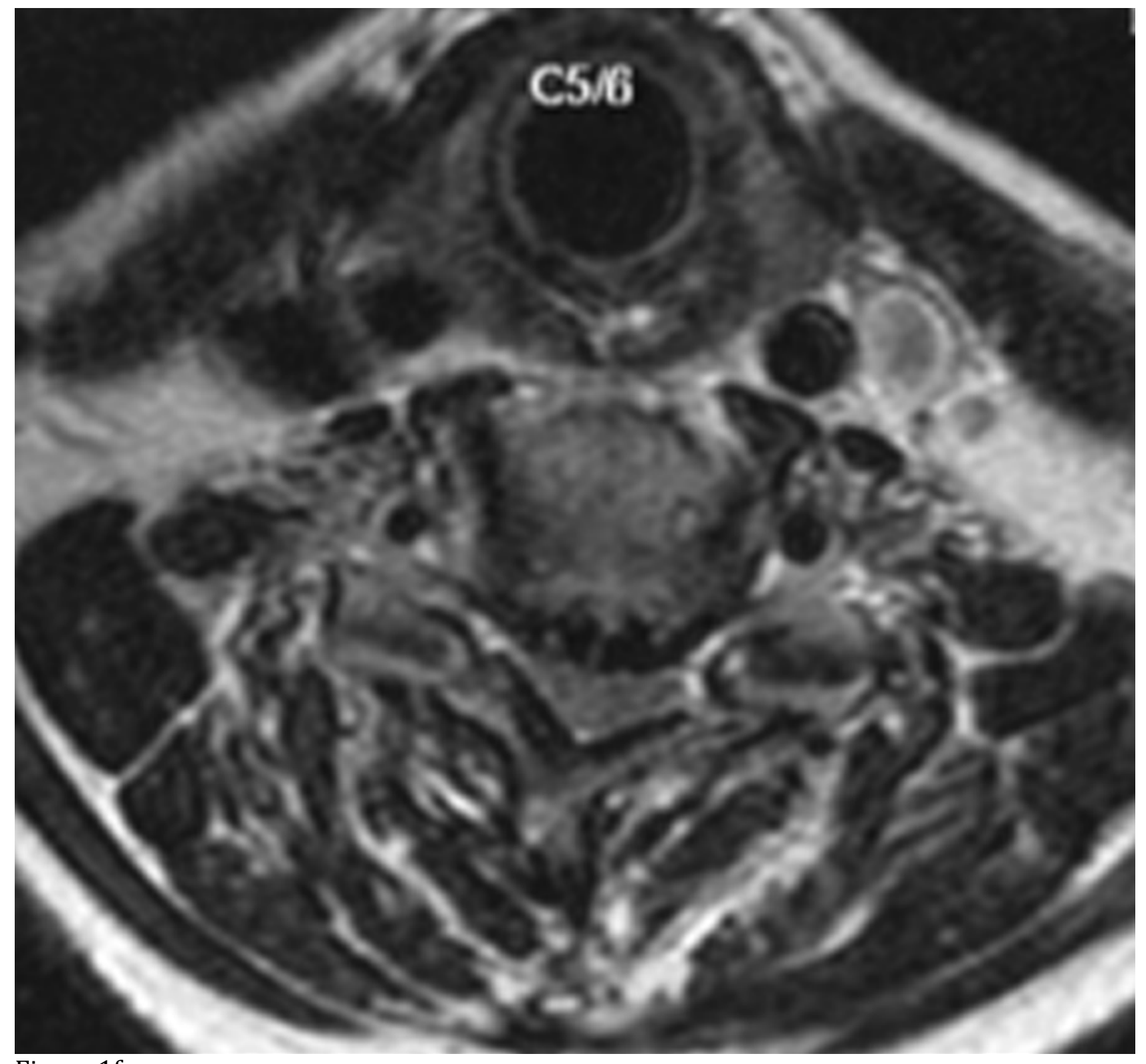

Figure 1f 


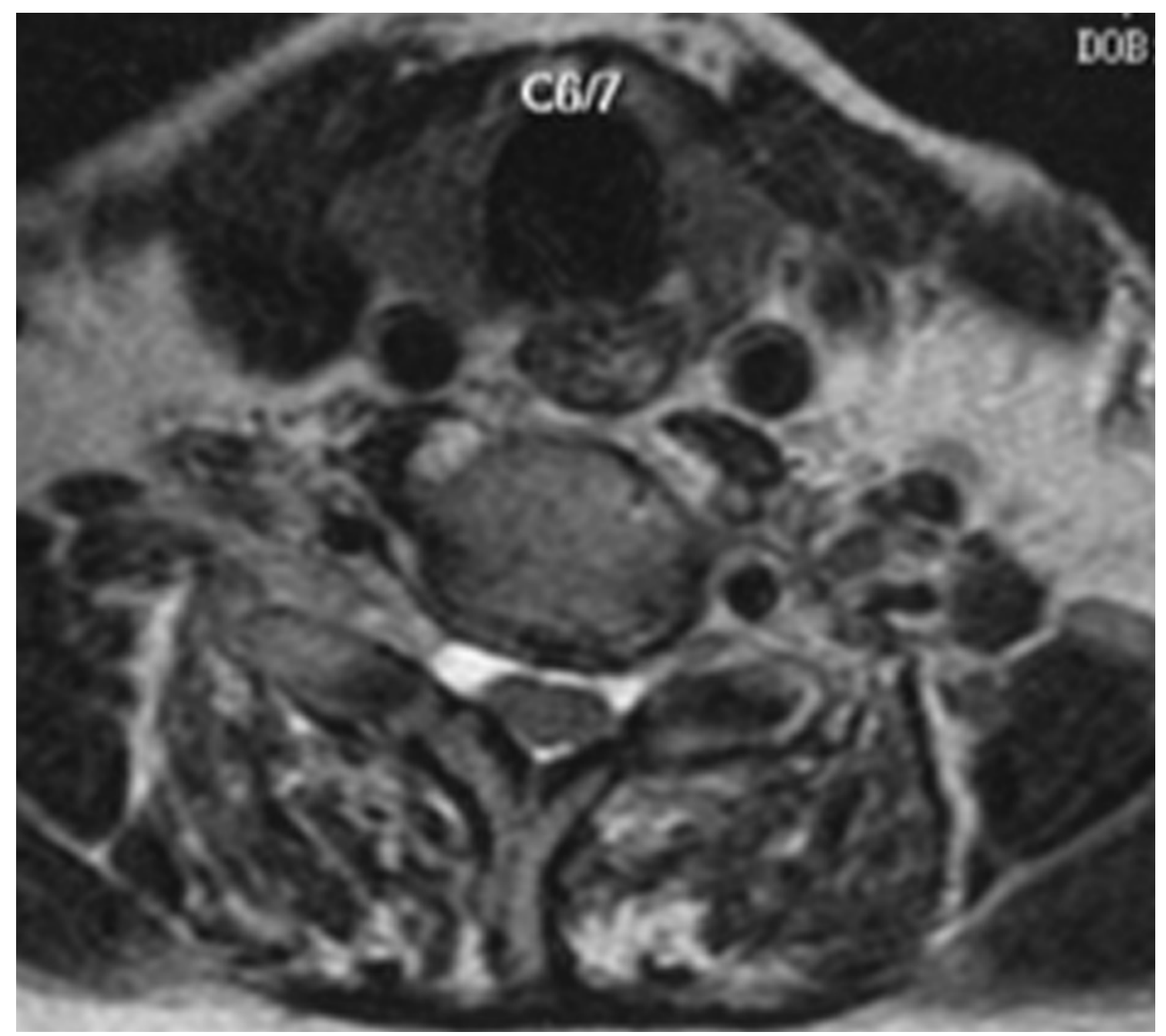

Figure 1g 


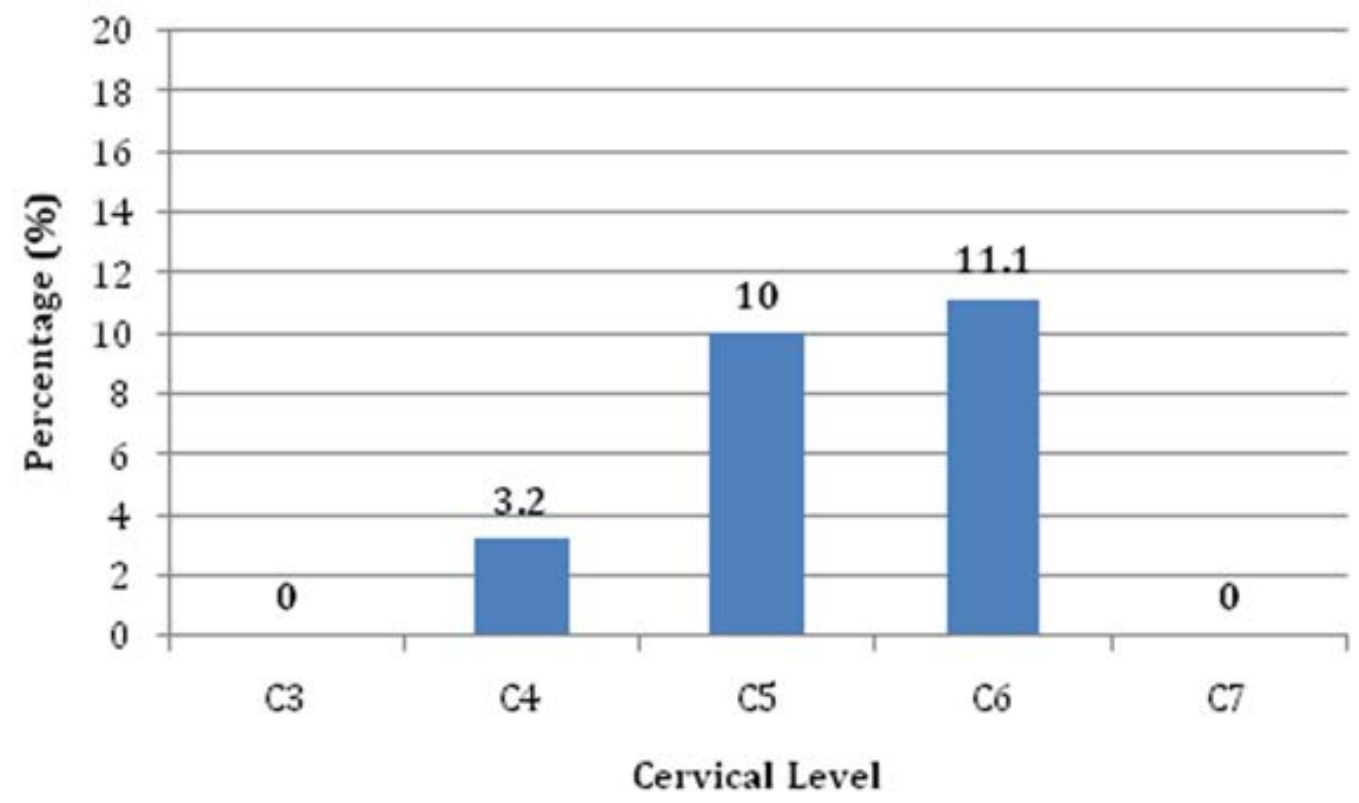

Figure 2 


\section{TABLES}

\begin{tabular}{||l||l||}
\hline $\begin{array}{l}\text { Partial Closure } \\
\text { - incomplete closure of an individual } \\
\text { lamina }\end{array}$ & $\begin{array}{l}\text { Segmental Closure } \\
\text { - a subset of the elevated laminas had } \\
\text { spring-back closure }\end{array}$ \\
\hline $\begin{array}{l}\text { Complete Closure } \\
\text { - complete closure of an individual } \\
\text { lamina, i.e. APD same as pre-operative or } \\
\text { less }\end{array}$ & $\begin{array}{l}\text { Total Construct Closure } \\
\text { - all elevated laminas had spring-back } \\
\text { closure }\end{array}$ \\
\hline
\end{tabular}

Table 1: Classification of "spring-back" closure. Though further combinations are possible it is limited by the supra- and inter-spinous ligaments of the intact spinous processes.

\begin{tabular}{cccccccc}
\hline \hline Case & $\begin{array}{c}\text { Age } \\
\text { (Years) }\end{array}$ & Sex & $\begin{array}{c}\text { Spring- } \\
\text { Back } \\
\text { Assessment } \\
\text { (months) }\end{array}$ & $\begin{array}{c}\text { Preoperative } \\
\text { JOA Score }\end{array}$ & $\begin{array}{c}\text { Postoperative } \\
\text { Best JOA } \\
\text { Score }\end{array}$ & $\begin{array}{c}\text { Postoperative } \\
\text { JOA Score After } \\
\text { Spring-Back }\end{array}$ & $\begin{array}{c}\text { Final Follow-up } \\
\text { JOA Score }\end{array}$ \\
\hline 1 & 48 & $\mathrm{M}$ & $6 \mathrm{~m}$ & 6 & 10 & 6 & 7.5 \\
2 & 77 & $\mathrm{~F}$ & $6 \mathrm{~m}$ & 12 & 13 & 12.5 & 13 \\
3 & 69 & $\mathrm{~F}$ & $6 \mathrm{~m}$ & 3 & 11 & 8.5 & 9 \\
\hline \hline
\end{tabular}

Table 2: Neurological outcome in patients with spring-back complication. (M=Male;

$\mathrm{F}=$ female) 